\title{
Efecto del tratamiento con leptina sobre la actividad del sistema glucosensor y la expresión de neuropéptidos implicados en la regulación de la ingesta de alimento en trucha arcoíris (Onchorhynchus mykiss)
}

\author{
Ariel J. Aguilar*
}

Recibido: octubre 2012/ Aceptado: noviembre 2012

La presente revisión se focaliza en el efecto modulatorio de la leptina sobre la actividad del sistema glucosensor y la expresión de neuropéptidos orexigénicos y anorexigénicos involucrados en la regulación de la ingesta de alimento en trucha arco iris. De manera similar al efecto que produce en mamíferos, la administración ICV de leptina induce cambios dosis dependiente en los parámetros relacionados con la glucosensibilidad en hipotálamo y cerebro posterior (incrementos del potencial glucolítico y glucogenogénico en coordinación con incrementos de la actividad de glucoquinasa y de los niveles de ARNm de genes involucrados en la respuesta glucosensora) compatible con lo observado en peces bajo condiciones de hiperglucemia, una situación que produce anorexia. La comprensión de la especificidad de la acción de la leptina en peces es menos clara que en mamíferos. Existen evidencias que sugieren que la acción central de la leptina sobre el sistema glucosensor está relacionada con la activación de la vía JAK/STAT e IRS-PI3K. Por otro lado, el tratamiento con leptina ICV incrementa el potencial glucogenolítico en hígado, el cual podría estar asociado con incrementos de la actividad simpática, y en hipotálamo induce decrecimiento de los niveles de ARNm de NPY. Por tanto, considerando la acción orexigénica del NPY en peces, pareciera que el efecto

Laboratorio de Fisiología Animal, Departamento de Biología, Facultad de Ciencia y Tecnología, Universidad Nacional Autónoma de Nicaragua, León, Nicaragua.

Correo electrónico:ajagui2002@yahoo.com 
anoréctico de la leptina puede estar mediado por la reducción de la expresión de NPY en el hipotálamo y que esos cambios pueden estar relacionados con la activación del sistema glucosensor.

Palabras clave: Sistema glucosensor, leptina, neuropéptidos, ingesta de alimentos, Onchorhynchus mykiss

\section{Glucosensibilidad}

Los peces teleósteos en general son incapaces de disminuir rápidamente los niveles de glucosa circundantes tras una carga de glucosa o tras la ingesta de alimento con altos niveles de carbohidratos, razón por la que se les considera intolerantes a la glucosa (Moon, 2001). El metabolismo de carbohidratos en los peces (especialmente en especies carnívoras) parece jugar un papel secundario al de los lípidos y las proteínas (Hemre et al., 2002). Sin embargo, los carbohidratos son claves para el metabolismo de todas las especies de peces, aunque no está clara su importancia como una vía energética en los tejidos. Entonces, ¿Por qué no regular el metabolismo de proteínas o lípidos en vez del metabolismo de la glucosa? La respuesta a esta pregunta no se sabe todavía. En mamíferos, la glucosa es la molécula combustible utilizada por la mayoría de los tejidos para liberar energía en condiciones normales, y cuando es necesario, la glucosa se sintetiza fácilmente a partir de moléculas no carbohidratos. Así, la glucosa es el sustrato preferido (si no obligatorio) usado por ciertos tejidos como el cerebro, retina, células rojas sanguíneas y médula adrenal como fuente de energía. En teleósteos, también hay evidencias del uso de la glucosa como principal sustrato energético en varios tejidos como en las células rojas en el pez cuervo (Hemitripterus americanus) (Sephton et al., 1991) o en el cerebro de trucha arco iris (Oncorhynchus mykiss) (Di Angelo et al., 1987). La glucosa es también el sustrato energético fundamental para el mantenimiento de varios tejidos en ciclóstomos como la lamprea (Petromyzon marinus) (Foster et al., 1993) y la lamprea del Atlántico (Mixine glutinosa) (Sidell et al., 1984).

Por tanto, en los peces los niveles de glucosa fluctúan en respuesta a cambios medioambientales, lo que junto con su necesidad para el funcionamiento de diferentes tejidos, incluido el cerebro (Soengas y Aldegunde, 2002), sugiere que la glucosa puede funcionar como señal metabólica en coordinación con las respuestas fisiológicas para mantener la homeostasis dentro del animal.

\subsection{Evidencias sobre la capacidad para detectar cambios en los niveles de glucosa en peces}

Varios estudios realizados en peces sugieren la posible existencia de glucosensores cerebrales debido a que: a) En trucha arco iris la hiperglucemia inducida experimentalmente conlleva a la disminución de la ingesta de alimento, mientras que el tratamiento ICV con 2-D-Glucosa (2-DG) incrementa la ingesta de alimento (Soengas y Aldegunde, 2004). b) Existen cambios paralelos entre los niveles de glucógeno cerebral, actividad y expresión de GKy cambios en los niveles de glucosa 
en plasma de trucha arco iris (Polakof et al., 2007a). c) Truchas arco iris realimentadas durante 7 días, tras 14 días de privación de alimento, muestran incrementos de los niveles de glucógeno y de la actividad y expresión de GK en el hipotálamo y cerebro posterior, así como incrementos en los niveles de glucosa plasmática (Soengas et al., 2006). d) El uso de la glucosa en el cerebro de Myoxocephalus scorpius se incrementa bajo condiciones hiperglucémicas (MacCormack et al., 2007). e) Existen cambios en los niveles de glucógeno y actividad y expresión de GK en el hipotálamo y cerebro posterior de trucha arco iris cuando se somete a cambios en los niveles de glucosa circulantes (Polakof et al., 2008a, b y c). Estos resultados son consistentes con el modelo conocido en las neuronas glucosensoras de los mamíferos (Marty et al).

Otro grupo de evidencias también sugieren la existencia de un sistema glucosensor vinculado con la secreción de insulina en las células $\square$ pancreáticas de los peces. Aunque los aminoácidos son los secretagogos más importantes de insulina en los peces (Navarro et al., 2002; Andoh, 2007), en casi todos los estudios realizados hasta la fecha, ya sean in vivo o in vitro, la glucosa ha sido capaz de estimular la liberación de insulina (Epple et al., 1987; Mommsen y Plisetskaya, 1991). Las evidencias que sugieren la existencia de un sistema glucosensor en el páncreas endocrino de los peces son: a) La presencia de GK en los cuerpos de Brockmann (BB) de Hippoglossus hippoglossus (Tranulis et al., 1997). b) El hecho de que la 2-DG y la manosa estimulan la secreción de insulina en el pez gato, Ictalurus punctatus (Ronner y Scarpa, 1987; Ronner, 1991). c) Los resultados obtenidos por Ronner y Scarpa (1987) de que el incremento de los niveles $\mathrm{K}^{+}$induce una pronunciada secreción de insulina en el pez gato. d) Cambios en la actividad y expresión de GK, expresión de GLUT2, y en los niveles de glucógeno en BB de trucha arco iris, cuando ésta es sometida a cambios en los niveles de glucosa (Polakof et al., 2007b).

\subsection{El cerebro de los peces como tejido consumidor de glucosa}

El cerebro de los peces puede utilizar otros combustibles además de la glucosa, tales como los cuerpos cetónicos, lactato, ácidos grasos y aminoácidos (Soengas y Aldegunde, 2002). Sin embargo, el cerebro tiene las mayores tasas de utilización de glucosa por unidad de peso, en todos los tejidos examinados en trucha arco iris, con valores tan altos como los descritos en ratas (Washburn et al., 1992). La principal diferencia a nivel del metabolismo de la glucosa entre los peces y los mamíferos es la enorme tolerancia de los peces a la hipoglucemia. Algunos teleósteos sobreviven luego de administrarles dosis farmacológicas de insulina, llegando incluso a niveles de glucosa en sangre casi indetectables, sin observarse ningún síntoma de trastorno nervioso (Mommsen y Plisetskaya, 1991). Sin embargo, cuando un pez activo que contiene niveles relativamente bajos de glucógeno en el cerebro, está hipoglucémico, se convulsiona y muere de un modo similar a la situación que ocurre en los mamíferos (Leibson, 1973). Para explicar este extraordinario fenómeno que ocurre en los peces, varios autores señalan que el carecer de efectos perjudiciales debido a la hipoglucemia se debe principalmente a su dependencia del metabolismo de lípidos y proteínas, y que los requerimientos de glucosa en los tejidos como el cerebro podrían ser excepcionalmente pequeños. Sin embargo, una importancia reducida del metabolismo de la glucosa en el cerebro entra en conflicto con los hallazgos 
en varios estudios que muestran cantidades importantes de glucógeno en el tejido cerebral, no solo en teleósteos sino también en ciclóstomos (Schmidt y Weneger, 1988; Foster et. Al., 1993; Plisetskaya et al., 1993), que ciertamente son mucho más altos que los descritos en otros vertebrados. En este sentido, cabe mencionar que la actividad de la glucosa 6-fosfatasa (G6Pasa) ha sido descrita en el cerebro de varias especies de peces (Rovainen, 1970; Plisetskaya et al., 1985; Polakof et al., 2008a). Por tanto, es posible que las reservas de carbohidratos en el cerebro le permitan a este tejido ser relativamente autónomo del aporte de glucosa sanguínea, especialmente durante alteraciones metabólicas de duración limitada.

La privación de alimento propicia la disminución de la glucemia en muchas especies de peces (Navarro et al., 1995). En el cerebro de los mamíferos, la hipoglucemia asociada con la privación de alimento mejora la captación de glucosa el primer día de ayuno, mientras que en períodos de tiempo más prolongados se da una disminución en la tasa de transporte de glucosa (Davson et al., 1996). Esta situación no ocurre en los peces ya que la inducción de hipoglucemia no altera la captación de glucosa (Blasco et al., 1996), sugiriendo que en el cerebro de los peces el metabolismo podría funcionar independientemente del sistema de transporte de glucosa. De hecho, la capacidad del cerebro de los salmónidos de utilizar la glucosa exógena disminuye durante la privación de alimento (Soengas et al., 1996, 1998). Es más, en salmónidos hipoglucémicos (inducidos por tratamiento con insulina o privación de alimento) los niveles de glucógeno disminuyen en el cerebro, apoyado por los cambios observados en el ratio de actividad de las enzimas GSasa/GPasa (Soengas et al., 1996, 1998; Polakof et al., 2007a, 2008a), lo que indica que en el cerebro el metabolismo del glucógeno local puede sostener la demanda de glucosa, independientemente de los cambios en los niveles de glucosa circulantes. Por ello, bajo condiciones de hipoglucemia severa o de privación de alimento durante largos períodos, la glucosa almacenada en el cerebro suple las necesidades del mismo. Este fenómeno también ha sido descrito en mamíferos, donde el glucógeno cerebral proporciona combustible durante períodos de tiempo en que los niveles de glucosa plasmática disminuyen (Choi et al., 2003).

Cabe mencionar que, tanto la trucha arco iris como los humanos tienen la capacidad de preservar los niveles de glucógeno en el cerebro tras situaciones de hipoglucemia (Choi et al., 2003; Soengas et al., 2006). Es posible que el cerebro, en peces y mamíferos, se adapte a alteraciones en la glucemia por medio de varios mecanismos (Choi et al., 2003), y uno de ellos podría ser los cambios en el metabolismo del glucógeno. En este sentido, aún cuando el cerebro de los peces muestra una independencia metabólica relativa sobre el sistema de abastecimiento sanguíneo en condiciones normales, el glucógeno cerebral juega el papel de neuroprotector in vivo. Por tanto, el posible mecanismo implicado en la iniciación de la disminución de los depósitos de glucógeno cerebral, puede ser debido a la capacidad cerebral de detectar los cambios de los niveles de glucosa circundantes a nivel local y sistémico. En este contexto, varios estudios en trucha arco iris, sugieren que los cambios en la glucemia se detectan en áreas discretas del cerebro, respondiendo con cambios en los niveles de glucógeno local (Soengas et al., 2006; Polakof et al., 2007b). Por consiguiente, en la trucha arco iris la hipoglucemia generaría un efecto mucho mayor que la hiperglucemia sobre el mantenimiento de los niveles de glucógeno en el cerebro 
(Soengas et al., 2006; Polakof et al., 2007b) aún a pesar de no existir cambios en la captación de glucosa por parte del cerebro en condiciones hiperglucémicas, tal y como se ha descrito en la trucha común (Salmo trutta) (Blasco et al., 1996).

\subsection{Componentes del sistema glucosensor a nivel central}

En los mamíferos los sensores de glucosa cerebrales son neuronas especializadas que responden a fluctuaciones en las concentraciones de glucosa con cambios en su frecuencia de descarga, incrementándola (neuronas excitables por glucosa, GE) o disminuyéndola (neuronas inhibibles por glucosa, GI) en respuesta a un incremento en los niveles de glucosa. El mecanismo de las neuronas GI se conoce solo parcialmente, mientras que está bastante bien caracterizado el de las neuronas GE (Marty et al., 2007). Para el funcionamiento de este mecanismo se precisa: a) Captar la glucosa por medio de un transportador de baja afinidad GLUT2. b) Fosforilar la glucosa por medio de la enzima glucoquinasa $(G K)$ para iniciar la glucólisis, e incrementar el ratio intracelular ATP/ADP. c) Lograr el cierre de los canales de potasio dependiente de ATP $\left(\mathrm{K}^{+}{ }_{\text {ATP }}\right)$ para facilitar la despolarización de la membrana y permitir la entrada del calcio $\left(\mathrm{Ca}^{2+}\right)$ a través de los canales de calcio tipo L (VDCC) con el subsiguiente incremento de la frecuencia de descarga (Marty et al., 2007).

Hasta el momento, en los peces, no existen evidencias directas sobre la presencia de neuronas excitables por glucosa (GE) o neuronas inhibibles por glucosa (GI). Se hipotetiza sin embargo su presencia en base a los cambios observados en la actividad y expresión de algunos componentes del sistema glucosensor, pero hay que tener en cuenta que en las áreas donde se dan las respuestas (hipotálamo y cerebro posterior) hay presencia de varias clases de neuronas. Hasta la fecha no existen estudios sobre los cambios en la frecuencia de descarga de las neuronas, para caracterizar su comportamiento en respuesta a las concentraciones de glucosa y de esta forma definir las áreas glucosensoras. Por otra parte, en mamíferos, los astrocitos hipotalámicos también detectan cambios en los niveles de glucosa (Blouet y Schwartz, 2010) por medio de la interacción con neuronas glucosensoras a lo que responden con la producción de lactato. En peces ésta capacidad glucosensora de la glía no ha sido evaluada todavía.

Muchos autores sugieren que la gluco detección podría realizarse independientemente de la presencia de GLUT2 (Kang et al., 2004), pero la isoforma GLUT2 probablemente esté implicada en la glucosensibilidad en los mamíferos (Thorens, 2001). GLUT2 se ha encontrado en el cerebro de varias especies de peces como el pez zebra (Danio rerio) (Castillo et al., 2009), trucha arco iris (Soengas et al., 2006; Polakof et al., 2007b) y lubina (Dicentrarchus labrax) (Terova et al., 2009). Se ha observado que la expresión de GLUT2 en el cerebro varía con la concentración de glucosa en el plasma de trucha arco iris (Polakof et al., 2007b), pero no en el bacalao (Gadus morhua) (Hall et al., 2006). Además, la expresión de GLUT2 ha sido caracterizada en áreas cerebrales discretas de trucha arco iris (Polakof et al., 2007b). Se han obtenido evidencias adicionales in vitro con respecto a la presencia de miembros de la familia de transportadores GLUT en el cerebro de los peces utilizando fármacos bloqueadores del transporte de glucosa, aunque esos trabajos no permiten discriminar entre las diferentes isoformas de GLUT (Polakof et al., 
2007c). La expresión de GLUT1 ha sido detectada en el cerebro de varias especies de peces tales como pez zebra (Jensen et al., 2006), bacalao (Hall et al., 2005), lubina (Terova., 2009) y tilapia (Oreochromis niloticus) (Hrytsenko et al., 2010), aunque no hay evidencias que apoyen su posible implicación en la glucosensibilidad, tal y como lo hace GLUT2. En un estudio reciente se ha observado que la expresión de ARNm de GLUT1 en el cerebro no cambia en respuesta a cambios en los niveles de glucosa (Hrytsenko et al., 2010).

Glucoquinasa $(\mathrm{GK})$ es una molécula fundamental para la glucosensibilidad y está considerada como el marcador glucosensor principal en los mamíferos (Magnuson et al., 2004; Kang et al., 2006). Se ha encontrado actividad y expresión GK en el cerebro de todos los vertebrados. En los mamíferos existen dos promotores alternativos para GK, los cuales son responsables de la iniciación de la transcripción de una manera mutuamente excluyente en distintos tejidos (Iynedjian, 2009). Los promotores "upstream" desencadenan la expresión de GK en tejidos no hepáticos, incluido el páncreas y el cerebro (Iynedjian, 2009). En peces, estudios realizados en dorada (Sparus aurata) revelan la presencia de un promotor de GK en el hígado (Egea et al., 2007).

La expresión génica de GK en el conjunto del cerebro ha sido estudiada en dos especies de peces como son la trucha arco iris (Panserat et al., 2000) y el pez zebra (González-Álvarez et al., 2009). Además, la actividad/expresión génica de GK se ha descrito en cinco áreas específicas del cerebro tales como el telencéfalo, cerebro posterior, cerebro medio, hipotálamo (Soengas et al., 2006) e hipófisis (Polakof, comunicación personal). Además, se ha descrito inmunoreactividad para GK en varias áreas hipotalámicas del cerebro de trucha arco iris (Polakof et al., 2009), incluyendo el hipotálamo lateral y núcleo tuberal lateral, anterior y posterior, que son áreas homólogas a las áreas donde se encuentran las neuronas glucosensoras en el hipotálamo de los mamíferos (Lynch et al., 2000). Es más, la inmunoreactividad para GK también se ha descrito en otras áreas fuera del hipotálamo (preóptica y núcleo oculomotor), donde el sistema glucosensor es probablemente funcional (Polakof et al., 2009). La inmunoreactividad para GK se ha visto no solamente en neuronas, sino también en tanacitos (Polakof et al., 2009) cuyo potencial capacidad glucosensora ha sido descrita en el cerebro de roedores (Rodríguez et al., 2005). En oposición a estos datos obtenidos en la especie carnívora trucha arco iris, en el pez omnívoro carpa común (Cyprinus carpio) la expresión de GK no se ha detectado en el cerebro (Panserat et al., 2000). La actividad y expresión de GK se incrementa en el hipotálamo y el cerebro posterior de trucha arco iris, cuando estos tejidos se someten a incrementos en la concentración de glucosa, ya sea in vivo o in vitro (Polakof et al., 2007 b y c). En el pez zebra, el incremento en la cantidad de carbohidratos en la dieta no produce ningún cambio en la expresión de GK al analizar toda la masa cerebral (González-Álvarez et al., 2009), pero este resultado no es sorprendente porque hay que considerar el efecto de la dilución que representa muestrear todo el tejido, lo que enmascara los posibles efectos localizados en áreas cerebrales específicas.

Los canales de potasio dependientes de ATP $\left(\mathrm{K}_{\text {ATP }}\right)$ juegan un papel fundamental en la glucosensibilidad, enlazando cambios entre el metabolismo de la glucosa con la actividad eléctrica de la membrana celular (Burdakov, 2007; Hibino et al., 2010). Casi no hay estudios que presenten evidencias sobre la presencia 
de canales $\mathrm{K}_{\mathrm{ATP}}$ en el cerebro de especies de no roedores. En el cerebro de varios teleósteos se expresan los dos componentes del canal $\mathrm{K}_{\mathrm{ATP}}$ denominados SUR y Kir, con secuencias muy similares a las encontradas en mamíferos tales como Kir6.2 y SUR1, incluyendo Kir6-like y SUR-like en la trucha arco iris (Polakof et al., 2008c), Kir8 en el salmón del atlántico (Salmo salar) (Leong et al., 2008; número de acceso en GenBank: NM_001140360) y Kir6.3 y SUR1 en el pez zebra (Zhang et al., 2006). Por otra parte se han obtenido evidencias indirectas utilizando fármacos que avalan la existencia de canales $K_{\text {ATP }}$ en el cerebro de trucha arco iris (Polakof et al., 2007c).

En los mamíferos, los genes que codifican los componentes del canal de calcio dependiente de potencial (VDCC) tipo L en las neuronas son Cav1.2 y Cav1.3 (Namkung et al., 2001). En los peces la presencia de este tipo de canal de calcio no ha sido estudiada completamente, y hasta la fecha, solamente se cuenta con la evidencia de que Cav1.2 y Cav1.3 se expresan en el cerebro del pez zebra (Sidi et al., 2004). Sin embargo, evidencias indirectas basadas en tratamientos farmacológicos con el inhibidor del canal tipo L nifedipina sugieren que ese isotipo del canal de calcio solo está activo parcialmente en la trucha arco iris (Polakof et al., 2007c).

\section{Posibles funciones relacionadas con el sistema glucosensor a nivel central}

\subsection{Regulación de la ingesta de alimento}

En los últimos años, utilizando trucha arco iris como modelo de pez teleósteo, se han realizado varios estudios que permiten sugerir que los cambios inducidos en la glucemia ya sean por medio de métodos farmacológicos o a través de dietas con diferentes concentraciones de carbohidratos, pueden modular la ingesta de alimento. En un primer estudio se observó una disminución de la ingesta de alimento tras haber inducido experimentalmente hiperglucemia, mientras que en truchas arco iris privadas de glucosa se observó un incremento de la ingesta de alimento (Soengas et al., 2004). En otros estudios, se ha observado que cuando trucha arco iris ha sido alimentada con dietas con un elevado contenido de carbohidratos o ha sido inyectada intraperitonealmente con glucosa, los niveles de glucosa plasmática se mantienen elevados, acompañados de la disminución de la ingesta de alimento, mientras que cuando se alimentan truchas con dietas sin carbohidratos o se inyectan con insulina, se observa una clara hipoglucemia acompañada de un incremento en la ingesta de alimento (Polakof et al., 2008a y b). Tanto en los estudios descritos a largo plazo, como en otros realizados a corto plazo (Soengas et al., 2006; Polakof et al., 2007b) está comprobado que el aumento de los niveles de glucosa en plasma (hiperglucemia) tiene efectos en algunas áreas cerebrales, (hipotálamo y cerebro posterior), por medio del incremento en la expresión y actividad GK, la acumulación de glucógeno y en algunos casos un aumento en el potencial glucolítico, mientras que las condiciones hipoglucémicas producen efectos contrarios. Estos resultados apoyan la hipótesis de que en respuesta a los elevados niveles de glucosa, el sistema glucosensor se activa en el cerebro de los peces y que tal activación probablemente modula la disminución de la ingesta de alimento, respuesta similar a la encontrada 
en aves (Denbow, 1999) y mamíferos (Seino et al., 2003; Marty et al., 2007). En trucha arco iris la expresión de los componentes del canal $\mathrm{K}_{\mathrm{ATP}}$ no se ve afectada por los altos niveles de glucosa (Polakof et al., 2008c), aunque evidencias farmacológicas sugieren que funciona como en los mamíferos (Polakof et al., 2007c). Es más, aunque esta función está todavía por ser explorada en otras especies de peces, los canales que contienen Kir6.3/SUR1 en el pez zebra (Zhang et al., 2006) pueden también estar implicados en tal regulación.

\section{2 ¿Cómo los cambios en los parámetros glucosensores se relacionan con cambios en la ingesta de alimento?}

En los mamíferos, las neuronas presentes en las áreas glucosensoras tienen la capacidad de producir péptidos implicados en el control de la ingesta de alimento (Schwartz et al., 2000; Blouet et al., 2010). Las neuronas del núcleo arcuato hipotalámico que producen neuropéptido $\mathrm{Y}(\mathrm{NPY})$ y proteína relacionada con agouti (AgRP) son neuronas inhibibles por glucosa (GI), mientras que las que producen pro-opiomelanocortina (POMC) y transcrito regulado por cocaína y anfetamina (CART) parecen ser neuronas excitables por glucosa (GE) (Dunn-Meynell et al., 2002). De este modo, cuando se incrementan los niveles de glucosa en plasma, aumenta la expresión de los péptidos POMC/CART y disminuye la expresión de los péptidos NPY/AgRP (Mobbs et al., 2005). En el cerebro de varias especies de peces se ha detectado ARNm de estos neuropéptidos en áreas análogas a las que están caracterizadas en mamíferos (Cerdá-Reverter et al., 2009) y la proteína GK está presente en las áreas cerebrales relacionadas con la regulación de la ingesta de alimento y la homeostasis energética, algunas de ellas homólogas a las que contienen las neuronas glucosensoras de los mamíferos tales como el núcleo ventromedial (VMN), arcuato (Arc), núcleo paraventricular (PVN) y el hipotálamo lateral (LH) en el hipotálamo (Polakof et al., 2009). Por tanto, se sabe que tanto GK como los péptidos relacionados con el control de la ingesta están presentes en estas áreas, lo que permite sugerir que hay una relación funcional entre ellos. En este sentido, un estudio reciente demostró que la expresión de varios neuropéptidos implicados en la regulación de la ingesta de alimento, en las áreas glucosensoras centrales (hipotálamo y cerebro posterior) de trucha arco iris, está regulada por cambios en la glucemia (Conde-sieira et al., 2010a) de un modo compatible con los efectos observados sobre la ingesta de alimento, tras realizar cambios en los niveles de glucosa en la misma especie (Polakof et al., 2008a y b). El cambio más importante fue la disminución de los niveles de ARNm de NPY y el incremento de los niveles de ARNm de CART y POMC en el hipotálamo de peces hiperglucémicos, mientras que en el cerebro posterior se incrementaron los niveles de ARNm de CART y CRF (Conde-sieira et al., 2010b). Todos estos cambios en la expresión de estos péptidos sugieren que la disminución de la ingesta de alimento observada en los peces bajo condiciones de estrés (Wendelaar Bonga, 1977) puede estar relacionada con la activación del sistema glucosensor por medio de cambios en la expresión de esos péptidos. El mecanismo a través del cual el estrés alteraría la ingesta de alimento por medio de la activación del sistema glucosensor no está muy claro todavía, pero un estudio reciente sugiere que alguno de los factores implicados en la activación del eje hipotalámico-hipofisiario- 
adrenal (HPI), como el Corticotropin release factor (CRF) o la corticotropina (ACTH), podrían estar implicados (Conde-sieira et al., 2012).

En los peces, como en otros vertebrados, el comportamiento alimentario está regulado por factores medioambientales y por complejos mecanismos homeostáticos que involucran factores hormonales periféricos y centrales y niveles de metabolitos presentes en la sangre (Volkoff et al., 2009). El principal regulador de la ingesta de alimento es el contenido energético presente en el alimento, ya que los peces comen casi exclusivamente hasta alcanzar un estatus energético óptimo (Boujard et al., 1994; Morales et al., 1994). Dado que los componentes mayoritarios de su dieta son las proteínas y los lípidos, no es sorprendente que elevados niveles de los mismos inhiban la ingesta (Gurure et al., 1995; Gelineau et al., 2001). Teniendo en cuenta la baja proporción relativa en la dieta, los carbohidratos deberían interactuar débilmente en la regulación de la ingesta de alimento. Sin embargo, varios estudios describen una disminución en la ingesta de alimento tras alimentar con dietas ricas en carbohidratos a especies de peces como la lubina (Peres et al., 2002; Moreira et al., 2008; Enes et al., 2010), trucha arco iris (Kaushik et al., 1989; Polakof et al., 2008b), salmón atlántico (Hemre et al., 1995) y diferentes especies de pez gato (Erfanullah et al., 1998). Ello avala la existencia de un mecanismo regulador de los niveles de glucosa sobre la ingesta de alimento.

El balance energético está regulado a través de múltiples vías implicadas en la estimulación (orexigénicas) o inhibición (anorexigénicas) del apetito, por lo que la regulación de la alimentación no involucra solamente al sistema nervioso central (SNC), sino que también involucra a otros tejidos periféricos como el tracto gastrointestinal, el tejido adiposo, el hígado, y el páncreas, aunque el hipotálamo es el tejido que juega un papel fundamental en la regulación de la ingesta de alimento y la homeostasis energética (Valassi et al., 2008; Volkoff et al., 2009).

En mamíferos, se sabe que los centros neuronales involucrados en el control del comportamiento alimentario, se encuentran en las áreas hipotalámicas VMN, DMN, PVN y LH (Kalra et al., 1999), específicamente el VMH se considera el área de la saciedad y el LH el área del hambre (Marty et al., 2007). En los peces no están completamente definidas las áreas que integran la saciedad o el hambre, pero estudios donde se han aplicado estímulos eléctricos al lóbulo inferior del hipotálamo, han demostrado que en este tejido se integra la respuesta de la ingesta de alimento (Demski, 1973; Roberts y Savage, 1978). Por tanto, la regulación del apetito y el peso corporal se produce por la interacción conjunta entre el cerebro y las señales que emanan de la periferia. Del resultado de esa integración, el cerebro, particularmente el hipotálamo, produce péptidos orexigénicos o anorexigénicos que participan en la regulación de la ingesta de alimento (Volkoff et al 2009).

Estudios recientes proporcionan evidencias sobre el papel modulatorio de diferentes péptidos implicados en la regulación de la ingesta de alimento sobre la actividad en algunas áreas glucosensoras en los peces, tales como leptina. Así, la administración central de leptina reduce los niveles de NPY en el hipotálamo y telencéfalo del carpín (Carassius auratus) (Volkoff et al., 2003) y en el cerebro de la carpa herbívora (Ctenopharyngodon idellus) (Li et al., 2010), mientras que en trucha arco iris, tanto la inyección intraperiteonal (IP) como intracerebroventricular (ICV) de leptina induce en el hipotálamo una reducción transitoria del ARNm de NPY 
(Murashita et al., 2008; Aguilar et. Al., 2010) y cuando solo es administrada IP produce una elevación de los niveles de ARNm de POMC (Murashita et al., 2008).

\section{Leptina}

Leptina es una hormona peptídica de $16 \mathrm{kDa}$, miembro de la clase-1 alfa de las citokinas helicoidales que en los mamíferos se expresan mayoritariamente en el tejido adiposo (Zhang et al., 1994) y en menor medida en otros tejidos como la placenta (Matsuzaki et al., 1997), cerebro y glándula mamaria (Smith-Kirwin, et al., 1998), músculo esquelético (Ahima y Flier, 2000), hipófisis (Morash et al., 1999) y estómago (Bado et al., 1998; Mix et al., 2000; Sobhani et al., 2000). Al péptido se le denomina la hormona de la obesidad, nombre que se otorga al gen que la codifica (ob). En vertebrados no mamíferos como el pollo se expresa principalmente en el hígado (Taouis et al., 1998), lo que sugiere que en vertebrados ancestrales el lugar original de producción de leptina podría haber sido el hígado.

El gen de la leptina ha sido identificado en peces (Kurokawa et al., 2005) y se encuentra leptina en adipocitos (Vegusdal et al., 2003) e hígado (Pfundt et al., 2009). Hay también evidencias de la expresión de receptores de leptina en tejidos como el músculo, la piel, las branquias, el cerebro y el hígado (Wong et al., 2007, Kurokawa y Murashita, 2009). Estudios en el salmón coho (Baker et al., 2000), pez gato (Silverstein y Plisetskaya, 2000) y pez verde (Londraville y Duvall, 2002) no reportan efectos del tratamiento con leptina sobre la ingesta de alimento o el peso corporal. Sin embargo, estudios en carpín (Volkoff et al., 2003; De Pedro et al., 2006) y trucha arco iris (Murashita et al., 2008; Kling et al., 2009) reportaron disminución de la ingesta de alimento tras tratamiento con leptina.

\subsection{Efecto de la leptina sobre el sistema glucosensor}

En peces, la leptina ejerce efecto sobre la modulación del sistema glucosensor, relacionados con los niveles de glucógeno en el hígado y el músculo así como los niveles circulantes de la hormona tras la administración intraperitoneal de la misma (Baker et al., 2000; De Pedro et al., 2006). Al administrar leptina ICV, en trucha arco iris, hay una activación de la glucogenolisis en el hígado (disminución de los niveles de glucógeno) acompañado de un ligero incremento del potencial gluconeogénico (Aguilar et al., 2010). Por tanto, dado que estos resultados son compatibles con los observados en hígado de peces sometidos a tratamientos con catecolaminas (Moon, 2004), sugerimos que la mayor presencia de leptina en el cerebro induce una respuesta en el metabolismo energético hepático, mediada por la activación del sistema nervioso simpático, resultando en un incremento de la producción de glucosa. Esta mayor producción, se debería principalmente al incremento de la glucogenolisis, en contraste con el modelo planteado para los mamíferos en el cual se debe fundamentalmente a un aumento de la gluconeogénesis (Pocai et al., 2005; Prodi y Obici, 2006). Tanto en experimentos in vivo como in vitro, en el sistema nervioso central leptina provoca aumento de la capacidad glucogenogénica (incremento en los niveles de glucógeno y actividad GSasa), mayor potencial glucolítico (incremento de 
la actividad PK y de los niveles de DHAP y GAP) y especialmente el incremento de la actividad GK en hipotálamo y cerebro posterior (Aguilar et al, 2010; Aguilar et al., 2011). Todos estos cambios indican la activación del sistema glucosensor, lo que se corresponde con la misma activación observada bajo condiciones de hiperglucemia (Polakof et al., 2008b y c) que propicia el efecto anoréctico de la leptina en esta misma especie (Murashita et al., 2008; Kling et al., 2009). Bajo este mismo contexto, estudios recientes muestran que la leptina administrada ICV ejerce efecto sobre la expresión de genes relacionados con la glucosensibilidad (GLUT2 y Kir.6-like), comparables con los observados en los parámetros metabólicos (Aguilar et al., 2010). Esto evidencia el papel de la leptina en la relación entre el sistema glucosensor y el control de la ingesta de alimento en peces.

En los mamíferos, el efecto de la leptina sobre la actividad glucosensora es dependiente de la concentración de glucosa (Morton et al., 2006). Igualmente en peces el tratamiento con leptina in vitro induce cambios en los parámetros implicados en la glucosensibilidad (Aguilar et al., 2011) similares a los descritos in vivo (Aguilar et al., 2010), que incluyen en hipotálamo y cerebro posterior incrementos en los niveles de glucosa, glucógeno y DHAP, así como incrementos en las actividades GK y GSasa cuando los tejidos son incubados en medios de cultivo con concentraciones de glucosa de 4 y $8 \mathrm{mM}$. En contraste, el tratamiento con leptina no produce efectos notorios cuando los tejidos se incuban en medio de cultivo con glucosa $2 \mathrm{mM}$ (Aguilar et al., 2011). Estos resultados son interesantes en relación al porqué los parámetros implicados en la glucosensibilidad no responden al tratamiento con la hormona cuando las concentraciones de glucosa son bajas, es decir bajo concentraciones de glucosa similares a las encontradas en plasma en condiciones de hipoglucemia. La administración de leptina en concentracion de $10 \mathrm{nM}$ en hipotalamo de trucha arco iris provoca cambios sobre los parámetros involucrados en la glucosensibilidad (Aguilar et al., 2011) similares a los observados en estudios in vitro en mamíferos (Wang et al., 2004). De la misma forma, al incubar tejido hipotalámico con tres concentraciones de glucosa $(2,4$ y $8 \mathrm{mM})$, dosis crecientes de leptina (10, 20 y 50 $\mathrm{nM}$ ) y tiempos diferentes de incubación (1 y 3h), para evaluar el efecto en las áreas glucosensoras de trucha arco iris, no sólo sugieren una activación de la expresión de GK con el incremento de la concentración de glucosa en el medio (activación del sistema glucosensor de acuerdo con estudios previos (Polakof et al., 2008b y c)) sino también que el tratamiento con leptina induce un incremento dosis creciente en la expresión de GK (Aguilar et al., 2011).

El efecto del tratamiento con leptina se revierte en presencia de agentes inhibidores de las vías de las proteínas JAK/STAT y IRS-PI(3)K sugiriendo la especificidad de la acción de la leptina. En general, se puede sugerir que leptina activa el sistema glucosensor y promueve la expresión de péptidos implicados en la ingesta de alimento (Aguilar et al 2010; Aguilar et al 2011) lo que permite reforzar la hipótesis de que los efectos de la leptina son debido a su acción directa y que estos no están relacionados a una acción indirecta de otro sistema hormonal (Aguilar et al., 2011). 


\subsection{Mecanismos de acción de la leptina en los sistemas glucosensores centrales}

El receptor de leptina pertenece a la familia de receptores de interleukina 6 de la clase 1 de receptores de citokinas. Los receptores de citokinas no tienen actividad enzimática intrínseca, sino que envían su señal a través de la Janus quinasa tipo 2 (JAK2) la cual induce la fosforilación de la tirosina en la unión con STAT3, STAT3 se dimeriza para convertirse en un factor de transcripción (Bates y Myers, 2003) y el conjunto es lo que se conoce como vía JAK/STAT. La leptina también activa la vía sustrato receptor de insulina-phosphatidylinositol-3-OK-kinase (IRS-PI3K). Así, la fosforilación de la proteína IRS sobre los residuos de tirosina es inducida por JAK2 y esto permite a la proteína IRS activar a la PI (3) K. Por conversión de PIP2 a PIP3, PI(3)K activa a PDK1 que inicia una serie de señales en múltiples enzimas incluyendo PKB y la PKC atípica (aPKC) (Morton et al., 2006).

En peces, existen evidencias respecto a algunos mecanismos específicos a través de los cuales la leptina ejerce su efecto sobre la función metabólica cerebral. Utilizando diferentes agentes inhibidores/antagonistas de distintas vías implicadas en la señalización de la leptina en mamíferos, se encontró que tanto in vivo como in vitro AG490, inhibidor de JAK2, produce en general una reversión de los efectos de la leptina en el cerebro posterior, aunque no tan claramente en el hipotálamo (Aguilar et al., 2011) lo que no coincide con los resultados obtenidos en mamíferos (Morton et al., 2009). Al igual que en mamíferos donde estudios recientes sugieren que, como la insulina, la leptina es también capaz de activar la vía IRS-PI3k (Robertson et al., 2008; Morton et al., 2009) en trucha arco iris Wortmannin (inhibidor de PI3K) claramente revierte la acción de la leptina en muchos de los parámetros evaluados en hipotálamo y cerebro posterior (Aguilar et al., 2010; Aguilar et al., 2011), denotando una vía similar a la descrita en mamíferos (Canabal et al., 2007a y b). Estos trabajos sugieren que una de las vías que podría usar la leptina para ejercer su efecto anoréctico está vinculada con las vías JAK/STAT e IRS-PI3K en cerebro posterior e IRS-PI3K en hipotálamo de trucha arco iris.

\subsection{Efecto de la leptina sobre la expresión de factores orexigénicos y anorexigénicos en áreas glucosensoras}

En hipotálamo y cerebro posterior de los mamíferos las neuronas glucosensoras contienen receptores de insulina, leptina y otros péptidos implicados en la homeostasis energética (Levin, 2006). Entre las neuronas que expresan receptores de leptina hay neuronas que coexpresan NPY y AgRP, y son inhibidas por la leptina, mientras que otras neuronas que coexpresan POMC y CART son estimuladas por la leptina (Duan et al., 2007; Morton et al., 2006; Wang et al., 2008). En trucha arco iris, al evaluar el efecto del tratamiento con leptina con concentraciones de glucosa (2, 4 y $8 \mathrm{mM})$ sobre la expresión de neuropéptidos en el hipotálamo y cerebro posterior, se observan dos tipos de respuestas diferentes. La respuesta más común es la ausencia de un efecto para el tratamiento con leptina sobre la expresión y la ausencia de este efecto no es modificada con la incubación del tejido con leptina en 
presencia de inhibidores de las vías JAK/STAT y IRS-PI3K (AG-490 o wortmannin). Este patrón se observó en el hipotálamo para la expresión de los neuropéptidos POMC, CART y CRF, y en el cerebro posterior para la expresión de NPY y POMC (Aguilar et al., 2011). Por lo tanto, en contraste con la respuesta observada en el modelo de los mamíferos, donde la expresión de POMC y CART se estimula por el tratamiento con leptina (Ambati et al., 2009; Mobbs et al., 2005), parece que la expresión de POMC y CART en hipotálamo y cerebro posterior de trucha arco iris no es sensible al tratamiento con leptina in vitro (Aguilar et al., 2011). En un estudio previo realizado in vivo, Murashita et al (2008) describieron un aumento transitorio en los niveles de ARNm de POMC en hipotálamo de trucha arco iris, tras tratamiento IP con leptina, lo que no se corresponde con lo encontrado in vitro por Aguilar et al (2011); sin embargo, en ese experimento se observo una tendencia al aumento de los niveles de ARNm de POMC en hipotálamos incubados con glucosa $8 \mathrm{mM}$ y teniendo en cuenta que ese estudio se llevó a cabo bajo condiciones in vitro, se puede sugerir que el efecto observado por Murashita et al., (2008) sobre la expresión de POMC podría ser atribuido a una acción indirecta de la leptina a través de cambios en otros sistemas endocrinos.

Por otro lado la expresión de NPY en hipotálamo de trucha arco iris desciende de un modo acusado en los grupos tratados con leptina comparados con los controles para las concentraciones de glucosa 2 y 4 mM (Aguilar et al., 2011). El efecto del tratamiento con leptina, inhibiendo la expresión de NPY coincide con evidencias obtenidas tanto en peces como en mamíferos. En peces, la administración ICV de leptina reduce los niveles de ARNm de NPY en el hipotálamo y telencéfalo del carpín (Volkoff et al., 2003), mientras que la inyección IP de leptina en la trucha arco iris induce un descenso en los niveles de ARNm de NPY en el hipotálamo (Murashita et al., 2008). En ratas, el tratamiento con leptina desciende la expresión del NPY en el hipotálamo (Ambati et al., 2009; Duan et al., 2007; Fekete et al., 2006), mientras que la deficiencia de leptina aumenta la expresión de este neuropéptido (Mobbs et al., 2005). En trucha arco iris, la especificidad de la acción de la leptina sobre la expresión del NPY se corrobora al comprobar los resultados tras tratamiento con leptina sola con los resultados tras tratamiento con leptina más los inhibidores de las vías JAK/ STAT y IRS-PI3K (Aguilar et al., 2010; Aguilar et al., 2011), lo que sugiere que las vías JAK/STAT e IRS-PI3K están implicadas en la acción de la leptina. Por tanto la respuesta a los inhibidores es similar a la observada en los parámetros glucosensores, sugiriendo que la acción de la leptina se lleva a cabo a través de esas vías y que está implicada en la regulación de los mecanismos glucosensores y el control de la ingesta de alimento, a través de los cambios en la expresión de los neuropéptidos. La participación de la leptina a través de esas vías podría ser similar a la que se presenta en los mamíferos, donde la vía JAK/STAT se activa por la leptina resultando en el descenso de la expresión de NPY (Tchaparian et al., 2010). Sin embargo, el hallazgo de que el tratamiento con leptina induce un marcado efecto en la expresión de NPY no es sorprendente ya que el NPY es el neuropéptido orexigénico principal en peces (Volkoff et al., 2009) y la acción inhibidora de la leptina en la ingesta de alimento se produce en parte por la inhibición en la expresión de NPY (Volkoff et al., 2003). Por otra parte, las acciones de la leptina en otros procesos fisiológicos en los peces no estrictamente relacionados con la ingesta de alimento tales como 
la función reproductiva, se sabe que también están mediadas por el NPY (Peyon et al., 2001, 2003). Es curioso que mientras el tratamiento con leptina provoca cambios en la respuesta glucosensora en hipotálamo y cerebro posterior, el efecto sobre la expresión de neuropéptidos se observa solo en el hipotálamo (Aguilar et al., 2011). Estos efectos difieren del modelo descrito en mamíferos donde los cambios en la expresión de dichos neuropéptidos ocurren en ambas áreas glucosensoras (hipotalamo y cerebro posterior) (Morton et al., 2006; Schwartz et al., 2000).

$\mathrm{Al}$ aplicar dosis crecientes de leptina $(10,20$ y $50 \mathrm{nM})$ en tiempos diferentes (1 y 3h), sobre la expresión de neuropéptidos en las áreas glucosensoras cerebrales (hipotalamo y cerebro posterior), no sólo sugieren una activación de la expresión de GK con el incremento de la concentración de glucosa en el medio (activación del sistema glucosensor de acuerdo con estudios previos (Polakof et al., 2008b y c)) sino también que el tratamiento con leptina induce un incremento dosis creciente en la expresión de GK, lo que concuerda con resultados obtenidos in vivo (Aguilar et al., 2011). El tratamiento con leptina induce un descenso en la expresión de NPY en hipotálamo que no es solamente dependiente de la concentración de leptina sino que también depende del tiempo, de modo que el mayor descenso en la expresión de NPY ocurrió tras tratamiento con la mayor concentración de leptina y con el mayor tiempo de incubación (Aguilar et al., 2011). El efecto de la leptina sobre la expresión de NPY ocurre con las 3 concentraciones de leptina usadas. A diferencia de los cambios observados en los niveles de ARNm de NPY, los otros neuropéptidos evaluados no muestran cambios en sus niveles de ARNm (POMC, CART y CRF en hipotálamo, y NPY y POMC en cerebro posterior). De esos neuropéptidos solo se observan cambios en los niveles de ARNm de POMC en la región hipotalámica, en la que se observa una tendencia aparente a aumentar con el tratamiento con leptina (Aguilar et al., 2011), sugiriendo que la expresión de POMC no es afectada por el tratamiento con leptina in vitro, en contraste con el modelo de los mamíferos (Ambati et al., 2009; Mobbs et al., 2005).

\section{Observación final}

Existen evidencias convincentes del posible papel de la leptina sobre la activación del sistema glucosensor periférico y central de trucha arco iris, provenientes de la demostración que al administrar leptina ICV hay un incremento de los niveles de glucosa plasmática, disminución de los niveles de glucógeno y aumento de la actividad de GPasa en hígado e incremento de los niveles de glucógeno y de la actividad de GK y GSasa en hipotalamo y cerebro posterior (Aguilar et al., 2010). Sin embargo, aunque se observa que la leptina ICV induce un descenso de la expresión de NPY que permite sugerir que al menos parte de la acción anoréctica de la leptina (mediada por los sistemas glucosensores) se debe a su efecto sobre la expresión de NPY, es necesario realizar otras investigaciones para dilucidar la escases de efectos observados tras tratamiento con leptina sobre la expresión de POMC y CART, que se sabe que también son neuropéptidos que participan en la regulación de la ingesta de alimento (Volkoff et al 2009; Volkoff and Peter, 2000; Murashita et al., 2008; Kling et al., 2009) debido a que el efecto de leptina sobre la expresión de 
estos neuropéptidos, en condiciones in vivo, puede ser mediada por la acción de otras señales hormonales. Igualmente el CRF es otro neuropéptido implicado en la regulación de la ingesta de alimento y aunque hasta la fecha no hay estudios en peces que relacionen a leptina con la expresión de CRF si se sabe que en condiciones de hiperglucemia concomitantemente se da la activación del sistema glucosensor central e incremento de la expresión de CRF (Conde-sieira et. Al., 2010).

\section{Referencias}

Aguilar, A.J., Conde-Sieira, M., Lopez-Patino, M.A., Miguez, J.M., Soengas, J.L. (2011). In vitro leptin treatment of rainbow trout hypothalamus and hindbrain affects glucosensing and gene expression of neuropeptides involved in food intake regulation. Peptides (32), 232-240.

Aguilar, A.J., Conde-Sieira,M., Polakof, S.,Miguez, J.M., Soengas, J.L. (2010). Central leptin treatment modulates brain glucosensing function and peripheral energy metabolism of rainbow trout. Peptides (31), 1044-1054.

Ahima, R.S. y Fliers, J.S. (2000). Leptin. Annu. Rev. Physiol. 62: 413-437.

Ambati, S., Duan, J., Choi, Y., Hartzell, D., Della-Fera, M. y Baile, C. (2009). ICV vs. VMH injection of leptin: Comparative effects on hypothalamic gene expression. Behav. Brain Res. (196), 279-285

Andoh, T. (2007). Amino acids are more important insulinotropins than glucose in a teleost fish, barfin flounder (Verasper moseri). Gen. Comp. Endocrinol. (151), 308-317.

Bado, A., Levasseur, S., Attoub, S., Kermorgant, S., Laigneau, J.-P., Bortoluzzi, M.N., Moizo, L., Lehy, T., Guerre-Millo, M., Le Marchand-Brustel, Y. y Lewin, M.J.M. (1998). The stomach is a source of leptin. Nature (394), 790-793.

Baker, D.M., Larsen, D.A., Swanson, P. y Dickhoff, W.W. (2000). Long-term peripheral treatment of immature coho salmon (Oncorhynchus kisutch) with human leptin has no clear physiologic effect. Gen. Comp. Endocrinol. (118), 134-138.

Bates, M. y Myers, M. (2003). The role of leptin receptor signaling in feeding and neuroendocrine function. Trends Endocrinol. Metabol. (14), 447-452.

Blasco, J., Fernández-Borrás, J., Marimon, I. y Requena, A. (1996). Plasma glucose kinetics and tissue uptake in brown trout in vivo: Effect of an intravascular glucose load. J. Comp. Physiol. B. (165), 534-541.

Blouet, C. y Schwartz, G.J. (2010). Hypothalamic nutrient sensing in the control of energy homeostasis. Behav. Brain Res. (209), 1-12.

Boujard, T. y Médale, F. (1994). Regulation of voluntary feed intake in juvenile rainbow trout fed by hand or by self feeders with diets containing 2 different protein energy ratios. Aquat. Living Resour. (7), 211-215.

Burdakov, D. (2007). $\mathrm{K}^{+}$channels stimulated by glucose: a new energy-sensing pathway. Pflugers Arch. (454), 19-27.

Canabal, D.D., Song, Z., Potian, J.G., Beuve, A., McArdle, J.J. y Routh, V.H. (2007a). Glucose, insulin and leptin signaling pathways modulate nitric oxide synthesis in glucose-inhibited neurons in the ventromedial hypothalamus. 
Am. J. Physiol. Regul. Integr. Comp. Physiol. (292), R1418-R1428.

Canabal, D.D., Potian, J.G., Duran, R.G., McArdle, J.J. y Routh, V.H. (2007b). Hyperglycemia impairs glucose and insulin regulation of nitric oxide production in glucose-inhibited neurons in the ventromedial hypothalamus. Am. J. Physiol. Regul. Integr. Comp. Physiol. (293), R592-600.

Castillo, J., Crespo, D., Capilla, E., Diaz, M., Chauvigne, F., Cerda, J. y Planas, J.V. (2009). Evolutionary structural and functional conservation of an ortholog of the GLUT2 glucose transporter gene (SLC2A2) in zebrafish. Am. J. Physiol. Regul. Integr. Comp. Physiol. (297), R1570-1581.

Cerdá-Reverter, J.M. y Canosa, L.F. (2009). Neuroendocrine systems of the fish brain. In: Bernier, N.J., Van der Kraak, G.J., Farrell, A.P., Brauner, C.J. (Eds.), Fish neuroendocrinology, Elsevier, Amsterdam, pp. 3-74

Choi, I.Y., Seaquist, E.R. y Gruetter, R. (2003). Effect of hypoglycemia on brain glycogen metabolism in vivo. J. Neurosci. Res. (72), 25-32.

Conde-Sieira, M., Aguilar, A.J., Lopez-Patiño, M.A., Miguez, J.M., Soengas, J.L., (2010a). Stress alters food intake and glucosensing response in hypothalamus, hindbrain, liver, and Brockmann bodies of rainbow trout. Physiol. Behav. (101), 483-493.

Conde-Sieira, M., Agulleiro, M., Aguilar, A.J., Miguez, J.M., Cerdá-Reverter, J. y Soengas, J.L. (2010b). Effect of different glycaemic conditions on gene expression of neuropéptidos involved in control of food intake in rainbow trout; interaction with stress. J. Exp. Biol. (213), 3858-3865.

Conde-Sieira, M., Alvarez, R., López-Patiño, M.A., Míguez, J.M., Flick, G y Soengas, J.L. (2012). ACTH-stimulated cortisol release from head kidney of rainbow trout is modulated by glucose concentration. J. Exp. Biol. (235), 3959-3972.

Davson, H., Segal, B. (1996). Physiology of the CSF and Blood brain barriers. CRC. Press: Boca Raton

De Pedro, N., Martínez-Álvarez, R. y Delgado, M.J. (2006). Acute and chronic leptin reduces food intake and body weight in goldfish (Carassius auratus). J. Endocrinol. (188), 513-20.

Demski, L.S. (1973). Feeding and aggressive behavior evoked by hypothalamic stimulation in a cichlid fish. Comp. Biochem. Physiol. A. (44), 685-692.

Denbow, D.M. (1999). Food intake regulation in birds. J. Exp. Zool. (283), 333-338.

DiAngelo, C.R. y Heath, A.G. (1987). Comparison of in vivo energy metabolism in the brain of rainbow trout, Salmo gairdneri and bullhead catfish, Ictalurus nebulosus during anoxia. Comp. Biochem. Physiol. B. (88), 297-303.

Duan, J., Choi, Y., Hartzell, D., Della-Fera, M.A., Hamrick, M. y Clifton, A. (2007). Effects of Subcutaneous Leptin Injections on hipothalamic Gene Profiles in Lean and ob/ob Mice. Obesity (15), 2624 -2633.

Dunn-Meynell, A.A., Routh, V.H., Kang, L., Gaspers, L. y Levin, B.E. (2002). Glucokinase is the likely mediator of glucosensing in both glucose-excited and glucose-inhibited central neurons. Diabetes (51), 2056-2065.

Egea, M., Meton, I. y Baanante, I.V. (2007). Sp1 and Sp3 regulate glucokinase gene transcription in the liver of gilthead sea bream (Sparus aurata). J. Mol. Endocrinol. (38), 481-492.

Enes, P., Sanchez-Gurmaches, J., Navarro, I., Gutierrez, J. y Oliva-Teles, A. (2010). 
Role of insulin and IGF-I on the regulation of glucose metabolism in European sea bass (Dicentrarchus labrax) fed with different dietary carbohydrate levels. Comp. Biochem. Physiol. A. (157), 346-353.

Epple, A., Brinn, J.E., Burggren, W., Ishii, S., Langer, H., Neuweiler, G. y Randall, D.J. (1987). The comparative physiology of the pancreatic islets. SpringerVerlag, Berlin.

Erfanullah, J.A.K. (1998). Effect of dietary carbohydrate-to lipid ratio on growth and body composition of walking catfish (Clarias batrachus). Aquaculture (161), 159-168.

Fekete, C. y Lechan, R.M. (2006). Neuroendocrine implications for the association between cocaine- and amphetamine regulated transcript (CART) and hypophysiotropic thyrotropin-releasing hormone (TRH). Peptides (27), 20122018.

Foster, G.D., Youson, J.H. y Moon, T.W. (1993). Carbohydrate metabolism in the brain of the adult lamprey. J. Exp. Zool. (267), 27-32.

González-Álvarez, R., Ortega-Cuellar, D., Hernández-Mendoza, A., Moreno-Arriola, E., Villasenor-Mendoza, K., Gálvez-Mariscal, A., Pérez-Cruz, M.E., MoralesSalas, I. y Velázquez-Arellano, A. (2009). The hexokinase gene family in the zebrafish: structure, expression, functional and phylogenetic analysis. Comp. Biochem. Physiol. B. (152), 189-195.

Gurure, R.M., Moccia, R.D. y Atkinson, J.L. (1995). Optimal protein requirements for young Artic charr (Salvelinus alpinus) fed practical diets. Aquaculture. Nutr. (1), 227-234.

Hall, J.R., Richards, R.C., MacCormack, T.J., Ewart, K.V. y Driedzic, W.R. (2005). Cloning of GLUT3 cDNA from Atlantic cod (Gadus morhua) and expression of GLUT1 and GLUT3 in response to hypoxia. Biochim. Biophys. Acta (1730), 245-252.

Hall, J.R., Short, C.E. y Driedzic, W.R. (2006). Sequence of Atlantic cod (Gadus morhua) GLUT4, GLUT2 and GPDH: developmental stage expression, tissue expression and relationship to starvation-induced changes in blood glucose. J. Exp. Biol. (209), 4490-4502.

Hemre, G.I., Mommsen, T.P. y Krogdahl, A. (2002). Carbohydrates in fish nutrition: effects on growth, glucose metabolism and hepatic enzymes. Aquaculture Nutr. (8), 175-194.

Hemre, G.I., Sandnes, K., Lie, Ø. y Waagbø, R. (1995). Blood chemistry and organ nutrient composition in Atlantic salmon, Salmo salar L., fed graded amounts of wheat starch. Aquaculture. Nutr. (1), 37-42.

Hibino, H., Inanobe, A., Furutani, K., Murakami, S., Findlay, I. y Kurachi, Y. (2010). Inwardly rectifying potassium channels: their structure, function, and physiological roles. Physiol. Rev. (90), 291-366.

Hrytsenko, O., Pohajdak, B., Xu, B.Y., Morrison, C., van Tol, B. y Wright, J.R. (2010). Cloning and molecular characterization of the glucose transporter 1 in tilapia (Oreochromis niloticus). Gen. Comp. Endocrinol. (165), 293-303.

Iynedjian, P.B. (2009). Molecular physiology of mammalian glucokinase. Cell. Mol. Life Sci. (66), 27-42.

Jensen, P.J., Gitlin, J.D. y Carayannopoulos, M.O. (2006). GLUT1 deficiency links 
nutrient availability and apoptosis during embryonic development. J. Biol. Chem. (281), 13382-13387.

Kalra, S.P., Dube, M.G., Pu, S., Xu, B., Horvath, T.L. y Kalra, P.S. (1999). Interacting appetite-regulating pathways in the hypothalamic regulation of body weight. Endocr. Rev.(20), 68-100.

Kang, L., Routh, V.H., Kuzhikandathil, E.V., Gaspers, L. y Levin, B.E. (2004). Physiological and molecular properties of rat hypothalamic ventromedial nucleus glucosensing neurons. Diabetes (53), 549-559.

Kang, L., Dunn-Meynell, A.A., Routh, V.H., Gaspers, L., Nagata, Y., Nishimura, T., Eikis, J., Zhang, B.B. y Levin, B.E. (2006). Glucokinase is a critical regulator of ventromedial hypothalamic neuronal glucosensing. Diabetes (55), 412-420.

Kaushik, S., Médale, F., Fauconneau, B. y Blanc, D. (1989). Effect of digestible carbohydrates on protein-Energy utilization and on glucose metabolism in rainbow trout (Salmo gairdneri R). Aquaculture (79), 63-74.

Kling, P., Ronnestad, I., Stefansson, S.O., Murashita, K., Kurokawa, T. y Björnsson, B.T. (2009). A homologous salmonid leptin radioimmunoassay indicates elevated plasma leptin levels during fasting of rainbow trout. Gen. Comp. Endocrinol. (162), 307-312.

Kurokawa, T., Uji, S. y Suzuki, T. (2005). Identification of cDNA coding for a homologue to mammalian leptin from pufferfish, Takifugu rubripes. Peptides (26), 745-750.

Kurokawa, T. y Murashita, K. (2009). Genomic characterization of multiple leptin genes and a leptin receptor gene in the Japanese medaka, Oryzias latipes. Gen. Comp. Endocrinol. (161), 229-237.

Leibson, L.G. (1973). Features of the metabolism and its endocrine regulation in fish with different motor activities. Evol. Physiol. Biochem. (8), 248-253.

Levin, B.E. (2006). Metabolic sensing neurons and the control of energy homeostasis. Physiol. Behav. (89), 486-489.

Li, G.G., Liang, X.F., Xie, Q., Li, G., Yu, Y. y Lai, K. (2010). Gene structure, recombinant expression and functional characterization of grass carp leptin. Gen. Comp. Endocrinol. (166), 117-127.

Londraville, R.L. y Duvall, C.S. (2002). Murine leptin injections increase intracellular fatty acid-binding protein in green sunfish (Lepomis cyanellus). Gen. Comp. Endocrinol. (129), 56-62.

Lynch, R.M., Tompkins, L.S., Brooks, H.L., Dunn-Meynell, A.A. y Levin, B.E. (2000). Localization of glucokinase gene expression in the rat brain. Diabetes (49), 693-700.

MacCormack, T.J. y Driedzic, W.R. (2007). The impact of hypoxia on in vivo glucose uptake in a hypoglycemic fish, Myoxocephalus scorpius Am. J. Physiol. Regul. Integr. Comp. Physiol. (292), R1033-R1042.

Magnuson, M.A. y Matschinsky, F.M. (2004). Glucokinase as a glucose sensor: past, present and future. In: Matschinsky, F.M., Magnuson, M.A. (Eds.), Glucokinase and glycemic disease: from basics tu novel therapeutics, vol. (16). Krager, Basel, pp. 1-17.

Marty, N., Dallaporta, M. y Thorens, B. (2007). Brain glucose sensing, counteregulation, and energy homeostasis. Physiology (22), 241-251. 
Matsuzaki, M., Milne, J., Aitken, R. y Wallace, J. (1997). Overnourishing pregnant adolescent ewes preserves perinatal fat deposition in their growth-restricted fetuses. Rep. Fert. Devel. (18), 357-364.

Mix, H., Widjaja, A., Jandl, O., Cornberg, M., Kaul, A., G€oke, M., Beil, W., Kuske, M., Brabant, G., Manns, M.P. y Wagner, S. (2000). Expression of leptin and leptin receptor isoforms in the human stomach. Gut (47), 481-486.

Mobbs, C.V., Isoda, F., Makimura, H., Mastaitis, J., Mizuno, T., Shu, I.W., Yen, K. y Yang, X.J. (2005). Impaired glucose signaling as a cause of obesity and the metabolic syndrome: the glucoadipostatic hypothesis. Physiol. Behav. (85), $2-23$.

Mommsen, T.P. y Plisetskaya, E.M. (1991). Insulin in fishes and agnathans: history, structure, and metabolic regulation. Rev. Aquat. Sci. (4), 225-259.

Moon TW. (2001). Glucose intolerance in teleost fish: fact or fiction? Comp. Biochem. Physiol. A. (129), 243-249.

Moon TW. (2004). Hormones and fish hepatocyte metabolism: the good, the bad and the ugly. Comp. Biochem. Physiol. B. (139), 335-45.

Morales, A.E., Cardenete, G., De la Higuera, M. y Sanz, A. (1994). Effects of dietary protein source on growth, feed conversion and energy utilization in rainbow trout, Oncorhynchus mykiss. Aquaculture (124), 117-126.

Morash, B., Li, A., Murphy, P.R., Wilkinson, M. y Ur, E. (1999). Leptin gene expression in the brain and pituitary gland. Endocrinology (140), 5995-5998.

Moreira, I.S., Peres, H., Couto, A., Enes, P. y Oliva-Teles, A. (2008). Temperature and dietary carbohydrate level effects on performance and metabolic utilisation of diets in European sea bass (Dicentrarchus labrax) juveniles. Aquaculture (274), 153-160.

Morton, G.J., Blevins, J.E., Kim, F., Matsen, M. y Figlewicz, D.P. (2009). The action of leptin in the ventral tegmental area to decrease food intake is dependent on Jak-2 signaling. Am. J. Physiol. Endocrinol. Metab. (297), E202-E210.

Morton, M.G., Cummings, D.E., Baskin, D.G., Barsh, G.S. y Schwartz, M.W. (2006). Central nervous system control of food intake and body weight. Nature (443), 289-295.

Murashita, K., Uji, S., Yamamoto, T., Ronnestad, I. y Kurokawa, T. (2008) Production of recombinant leptin and its effects on food intake in rainbow trout (Oncorhynchus mykiss). Comp. Biochem. Physiol. B. (150), 377-384.

Namkung, Y., Skrypnyk, N., Jeong, M.J., Lee, T., Lee, M.S., Kim, H.L., Chin, H., Suh, P.G., Kim, S.S. y Shin, H.S. (2001). Requirement for the L-type Ca(2+) channel alpha(1D) subunit in postnatal pancreatic beta cell generation. J. Clin. Invest. (108), 1015-1022.

Navarro, I. y Gutiérrez, J. (1995). Fasting and starvation. In: Hochachka, P.W., Mommsen, T.P. (Eds.), Biochemistry and Molecular Biology of Fishes, vol. Vol. (4). Elsevier, New York, pp. 394-434

Navarro, I., Rojas, P., Capilla, E., Albalat, A., Castillo, J., Montserrat, N., Codina, M. y Gutiérrez, J. (2002). Insights into insulin and glucagon responses in fish. Fish Physiol. Biochem. (27), 205-216.

Panserat, S., Blin, C. y Médale, F. (2000). Molecular cloning tissue distribution and sequence analysis of complete glucokinase cDNAs from gilthead seabream 
(Sparus aurata), rainbow trout and common carp. Biochim. Biophys. Acta (1474), 61-69.

Peres, H. y Oliva-Teles, A. (2002). Utilization of raw and gelatinized starch by European sea bass (Dicentrarchus labrax) juveniles. Aquaculture (205), 287-299.

Peyon, P., Zanuy, S. y Carrillo, M. (2001). Action of leptin on in vitro luteinizing hormone release in the european sea bass (Dicentrarchus labrax). Biol. Reprod. (65), 1573-1578.

Peyon, P., Vega-Rubín de Celis, S., Gómez-Requeni, P., Zanuy, S., Pérez-Sánchez, J. y Carrillo, M. (2003). In vitro effect of leptin on somatolactin release in the European sea bass (Dicentrarchus labrax): dependence on the reproductive status ad interaction with NPY and GnRH. Gen. Comp. Endocrinol. (132), 284-292.

Pfundt, B., Sauerwein, H. y Mielenz, M. (2009). Leptin mRNA and Protein Immunoreactivity in Adipose Tissue and Liver of Rainbow Trout (Oncorhynchus mykiss) and Immunohistochemical Localization in Liver. Anat. Histol. Embryol. (38), 406-410.

Plisetskaya, E.M., Foreman, R.E., Gorbman, A., Dodd, J.M. y Olson, R. (1985). Some aspects of hormonal regulation of metabolism in agnathans. In: Evolutionary biology of primitive fishes, Plenum, New York, pp. 339-361

Plisetskaya, E.M. y Mommsen, T.P. (1996). Glucagon and glucagon-like peptides in fishes. Intl. Rev. Cytol. (168), 187-257.

Pocai, A., Morgan, K., Buettner, C., Gutierrez-Juarez, R., Obici, S. y Rossetti, L. (2005). Central leptin acutely reverses diet-induced hepatic insulin resistance. Diabetes (54), 3182-3189.

Polakof, S., Ceinos, R.M., Fernández-Durán, B., Míguez, J.M. y Soengas, J.L. (2007a). Daily changes in parameters of energy metabolism in brain of rainbow trout: dependence on feeding. Comp. Biochem. Physiol. A. (146), 265-273.

Polakof, S., Míguez, J.M., Moon, T.W. y Soengas, J.L. (2007b). Evidence for the presence of a glucosensor in hypothalamus, hindbrain, and Brockmann bodies of rainbow trout. Am. J. Physiol. Regul. Integr. Comp. Physiol. (292), R1657-R1666.

Polakof, S., Míguez, J.M. y Soengas, J.L. (2007c). In vitro evidences for glucosensing capacity and mechanisms in hypothalamus, hindbrain, and Brockmann bodies of rainbow trout. Am. J. Physiol. Regul. Integr. Comp. Physiol. (293), R1410-R1420.

Polakof, S., Míguez, J.M. y Soengas, J.L. (2008a). Changes in food intake and glucosensing function of hypothalamus and hindbrain in rainbow trout subjected to hyperglycemic or hypoglycemic conditions. J. Comp. Physiol. A. (194), 829-839.

Polakof, S., Míguez, J.M. y Soengas, J.L. (2008b). Dietary carbohydrates induce changes in glucosensing capacity and food intake in rainbow trout. Am. J. Physiol. Regul. Integr. Comp. Physiol. (295), R478-R4789.

Polakof, S., Panserat, S., Plagnes-Juan, E. y Soengas, J.L. (2008c). Altered dietary carbohydrates significantly affect gene expression of the major glucosensing components in Brockmann bodies and hypothalamus of rainbow trout. Am. J. Physiol. Regul. Integr. Comp. Physiol. (295), R1077-R1088. 
Polakof, S., Rodriguez-Alonso, M. y Soengas, J.L. (2009). Immunohistochemical localization of glucokinase in rainbow trout brain. Comp. Biochem. Physiol. A. (153), 352-358.

Prodi, E. y Obici, S. (2006). Minireview: The Brain as a Molecular Target for diabetic therapy. Endocrinology (147), 2664-2669

Roberts, M.G. y Savage, G.E. (1978). Effects of hypothalamic lesions on the food intake of the goldfish (Carassius auratus). Brain Behav. Evol. (15), 150-164.

Robertson, S.A., Leinninger, G.M. y Myers, M.G. (2008). Molecular and neural mediators of leptin action. Physiol. Behav. (94), 637-42.

Rodríguez, E.M., Blázquez, J.L., Pastor, F.E., Peláez, B., Pena, P., Peruzzo, B. y Amat, P. (2005). Hypothalamic tanycytes: a key component of brain-endocrine interaction. Int. Rev. Cytol. (247), 89-164.

Ronner, P. (1991). 2-Deoxyglucose stimulates the release of insulin and somatostatin from the perfused catfish pancreas. Gen. Comp. Endocrinol. (81), 276-283.

Ronner, P. y Scarpa, A. (1987). Secretagogues for pancreatic hormone release in the channel catfish. Gen. Comp. Endocrinol. (65), 354-362.

Rovainen, C.M. (1970). Glucose production by lamprey meninges. Science (167), 889-890.

Schmidt, H. y Wegener, G. (1988). Glycogen phosphorylase in fish brain (Carassius carassius) during hypoxia. Biochem. Soc. Trans. (16), 621-622.

Schwartz, M.W., Woods, S.C., Porte Jr, D., Seeley, R.J. y Baskin, D.G. (2000). Central nervous system control of food intake. Nature (404), 661-671.

Seino, S. y Miki, T. (2003). Physiological and pathophysiological roles of ATPsensitive $\mathrm{K}^{+}$channels. Prog. Biophys. Mol. Biol. (81), 133-176.

Sephton, D.H., Macphee, W.L. y Driedzic, W.R. (1991). Metabolic enzyme activities, oxygen consumption and glucose utilization in sea raven (Hemitripterus americanus) erythrocytes. J. Exp. Biol. (159), 407-418.

Sidell, B.D., Stowe, D.B. y Hansen, C.A. (1984). Carbohydrate is the preferred metabolic fuel of the hagfish (Myxine glutinosa). Physiol. Zool. (57), 266-273.

Sidi, S., Busch-Nentwich, E., Friedrich, R., Schoenberger, U. y Nicolson, T. (2004). gemini encodes a zebrafish L-type calcium channel that localizes at sensory hair cell ribbon synapses. J. Neurosci. (24), 4213-4223.

Silverstein, J.T. y Plisetskaya, E.M. (2000). The effects of NPY and insulin on food intake regulation in fish. Am. Zool. (40), 296-308.

Smith-Kirwin, S.M., O’Connor, D.M., De Johnston, J., Lancey, E.D., Hassink, S.G. y Funanage, V.L. (1998). Leptin expression in human mammary epithelial cells and breast milk. J. Clin. Endocrinol. Metab. (83), 1810-1813.

Sobhani, I., Bado, A., Vissuzaine, C., Buyse, M., Kermorgant, S., Laigneau, J.P., Attoub, S., Lehy, T., Henin, D., Mignon, M. y Lewin, M.J. (2000). Leptin secretion and leptin receptor in the human stomach. Gut (47), 178-183.

Soengas, J.L. y Aldegunde, M. (2002). Energy metabolism of fish brain. Comp. Biochem. Physiol. B. (131), 271-296.

Soengas, J.L. y Aldegunde, M. (2004). Brain glucose and insulin: Effects on food intake and brain biogenic amines of rainbow trout. J. Comp. Physiol. A. (190), 641-649.

Soengas, J.L., Polakof, S., Chen, X., Sangiao-Alvarellos, S. y Moon, T.W. (2006). 
Glucokinase and hexokinase expression and activities in rainbow trout tissues: changes with food deprivation and refeeding. Am. J. Physiol. Regul. Integr. Comp. Physiol. (291), R810-R821.

Soengas, J.L., Strong, E.F. y Andrés, M.D. (1998). Glucose, lactate, and betahydroxybutyrate utilization by rainbow trout brain: changes during food deprivation. Physiol. Zool. (71), 285-293.

Soengas, J.L., Strong, E.F., Fuentes, J., Veira, J.A.R. y Andrés, M.D. (1996). Food deprivation and refeeding in Atlantic salmon, Salmo salar. Effects on brain and liver carbohydrate and ketone bodies metabolism. Fish Physiol. Biochem. (15), 491-511.

Tchaparian, E., Marshal, L., Cutler, G., Bauerly, K., Chowanadisai, W., Satre, M., Harris, C. y Rueker, R. (2010). Identification of transcriptional networks responding to pyrroloquinoline quinone dietary supplementation and their influence on thioredoxin expression, and the JAK/STAT and MAPK pathways. J. Biochem. (429), 515-526.

Taouis, M., Chen, J.-W., Daviaud, C., Dupont, J., Derouet, M. y Simon, J. (1998). Cloning the chicken leptin gene. Gene (208), 239-242.

Terova, G., Rimoldi, S., Brambilla, F., Gornati, R., Bernardini, G. y Saroglia, M. (2009). In vivo regulation of GLUT2 mRNA in sea bass (Dicentrarchus labrax) in response to acute and chronic hypoxia. Comp. Biochem. Physiol. B. (152), 306-316.

Thorens, B. (2001). GLUT2 in pancreatic and extra-pancreatic gluco-detection (review). Mol. Membr. Biol. (18), 265-273.

Tranulis, M.A., Christophersen, B. y Borrebaek, B. (1997). Glucokinase in Atlantic halibut (Hippoglossus hipoglossus) Brockmann bodies. Comp. Biochem. Physiol. B. (116), 367-370.

Valassi, E., Scacchi, M. y Cavagnini, F. (2008). Neuroendocrine control of food intake. Nutr. Metab. Cardiovasc. Dis. (18), 158-168.

Vegusdal, A., Sundvold, H., Gjoen, T. y Ruyter, B. (2003). An in vitro method for studying the proliferation and differentiation of Atlantic salmon preadipocytes. Lipids (38), 289-296.

Volkoff, H., Unniappan, S., Kelly, S.P. (2009). The endocrine regulation of food intake. In: Bernier NJ, Van der Kraak G, Farrell AP, Brauner CJ, editors. Fish neuroendocrinology. Amsterdam: Academic Press; (2009). p. 421-65.

Volkoff, H., Eykelbosh, A.J. y Peter, R.E. (2003). Role of leptin in the control of feeding of goldfish Carassius auratus: interactions with cholecystokinin, neuropeptide $\mathrm{Y}$ and orexin A, and modulation by fasting. Brain Res. (972), 90-109.

Volkoff, H. y Peter, R.E. (2000). Effects of CART peptides on food consumption, feeding and associated behaviors in the goldfish, Carassius auratus: actions on neuropeptide $Y$ and orexin A-induced feeding. Brain Res. (887), 125-133.

Wang, J.H., Wang, M., Yang, F., Yu, W., Wu, J., Liu, L., Caia, F. y Chen, J. (2008). Leptin regulated calcium channels of neuropeptide y and proopiomelanocortin neurons by activation of different signal pathways. Neuroscience (156), 89-98

Wang, R., Liu, X., Hentges, S.T., Dunn-Meynell, A.A., Levin, B.E., Wang, W. y Routh, V.H. (2004). The regulation of glucose-excited neurons in the hypothalamic 
arcuate nucleus by glucose and feeding-relevant peptides. Diabetes (53), 1959 1965.

Washburn, B.S., Bruss, M.L., Avery, E.H. y Freedland, R.A. (1992). Effects of estrogen on whole animal and tissue glucose use in female and male rainbow trout. Am. J. Physiol. Regul. Integr. Comp. Physiol. (263), R1241-R1247.

Wong, M., Yu, R., Ng, P., Law, S., Tsang, A. y Kong, R. (2007). Characterization of a hypoxiaresponsive leptin receptor (omLepR(L)) cDNA from the marine medaka (Oryzias melastigma). Mar Pollut Bull. (54), 797-803.

Zhang, C., Miki, T., Shibasaki, T., Yokokura, M., Saraya, A. y Seino, S. (2006). Identification and characterization of a novel member of the ATP-sensitive $\mathrm{K}^{+}$ channel subunit family, Kir6.3, in zebrafish. Physiol. Genomics (24), 290-297.

Zhang, Y., Proenca, R., Maffei, M., Barone, M., Leopold, L. y Friedman, J.M. (1994). Positional cloning of the mouse obese gene and its human homologue. Nature (372), 425-432. 